\title{
Aggressive G-CSF-producing gastric cancer complicated by lung and brain abscesses, mimicking metastases
}

\author{
Taku Yokoyama ${ }^{1}$, Masanobu Hyodo ${ }^{1}$, Yoshinori Hosoya ${ }^{1}$, Koji Koinuma ${ }^{1}$, Kentarou Kurashina ${ }^{1}$, \\ Shin Saitoh ${ }^{1}$, Yuuki Hirashima ${ }^{1}$, Wataru Arai ${ }^{1}$, Toru Zuiki ${ }^{1}$, Yoshikazu Yasuda ${ }^{1}$, Hideo Nagai ${ }^{1}$, \\ Nobuyuki Kanai ${ }^{2}$, and Ken SaItoh ${ }^{2}$ \\ ${ }^{1}$ Department of Surgery, Jichi Medical School, Minami-Kawachi, Tochigi 329-0498, Japan \\ ${ }^{2}$ Department, Pathology, Jichi Medical School, Tochigi, Japan
}

\begin{abstract}
A 57-year-old Japanese man had type II c gastric cancer with marked lymph node metastases associated with leukocytosis and elevated granulocyte colony-stimulating factor (G-CSF). Total gastrectomy and distal pancreatectomy with lymph node dissection were performed. Although the primary lesion was negative for G-CSF by histopathological immunostaining, a highly increased G-CSF m-RNA level, measured using reverse transcriptase-polymerase chain reaction in frozen sections, led to a diagnosis of G-CSF-producing gastric cancer. The leukocytes and G-CSF decreased immediately after surgery. He then had an intraabdominal recurrence, and was diagnosed with multiple tumors in his lung and brain, with abnormally elevated leukocytes and greatly increased G-CSF; he died 4 months after the surgery. Autopsy showed intraabdominal recurrence of cancer, with no metastases to the lung or brain, but with multiple brain and lung abscesses. We speculate that the excessively increased neutrophils induced by G-CSF infiltrated the lung and brain and formed abscesses, mimicking metastases.
\end{abstract}

Key words G-CSF · Gastric cancer · Abscess

\section{Introduction}

Gastric cancer that produces granulocyte colonystimulating factor (G-CSF) is thought to be an extremely rare disease with a poor prognosis. Although more than 20 cases of G-CSF-producing gastric cancer have been reported, mainly from Japanese institutes [1], little is known about the clinical course related to leukocytosis, owing to its rarity. Recently, we encountered a patient with G-CSF-producing stomach cancer with marked lymph node metastases treated with surgery, but he had an intraabdominal recurrence and died 4

Offprint requests to: $\mathrm{M}$. Hyodo

Received: March 7, 2005 / Accepted: April 11, 2005 months after the surgery, with abnormally elevated leukocyte levels induced by G-CSF. Autopsy revealed lung and brain abscesses, which had been clinically evaluated as distant metastases.

\section{Case report}

The patient was a 57-year-old Japanese man who had no remarkable past or family history. He was referred to our hospital because of fever, malaise, and anorexia, and was consequently hospitalized. On physical examination, his temperature was $38.2^{\circ} \mathrm{C}$, and there was no palpable abdominal mass. Blood biochemistry tests showed a high leukocyte level, of $34000 / \mathrm{mm}^{3}$ (90\% neutrophils). The tumor markers carcinoembryonic antigen (CEA) and carbohydrate antigen (CA)19-9 were not elevated, but the levels of G-CSF were high, at $107 \mathrm{pg} /$ $\mathrm{ml}(5.78-27.5 \mathrm{pg} / \mathrm{ml})$. Endoscopic examination showed type II c gastric cancer [2] in the posterior wall of the lower stomach, with extramural compression of the lesser curvature by lymph node metastasis. Stomach biopsy revealed the proliferation of large, multinucleated cells, which led to a diagnosis of undifferentiated carcinoma. Abdominal computed tomography (CT) showed a 7-cm lymph node lesion at the lesser curvature.

After admission, the patient developed a high fever (temperature of $39^{\circ} \mathrm{C}$ ) and his sensation of general fatigue worsened. The leukocyte level rose to $67000 / \mathrm{mm}^{3}$. A second endoscopy, on the eighteenth hospital day, revealed enlargement and ulceration of the mass on the lesser curvature, and an operation was performed on the twentieth day after admission. During surgery, the tumor was evaluated as T1 (submucosal invasion), N3 (metastasis to the posterior side of the common hepatic artery), H0 (without liver metastasis), and P0 (without peritoneal dissemination), stage IV [2]. Total gastrectomy plus distal pancreatectomy with lymph node dis- 


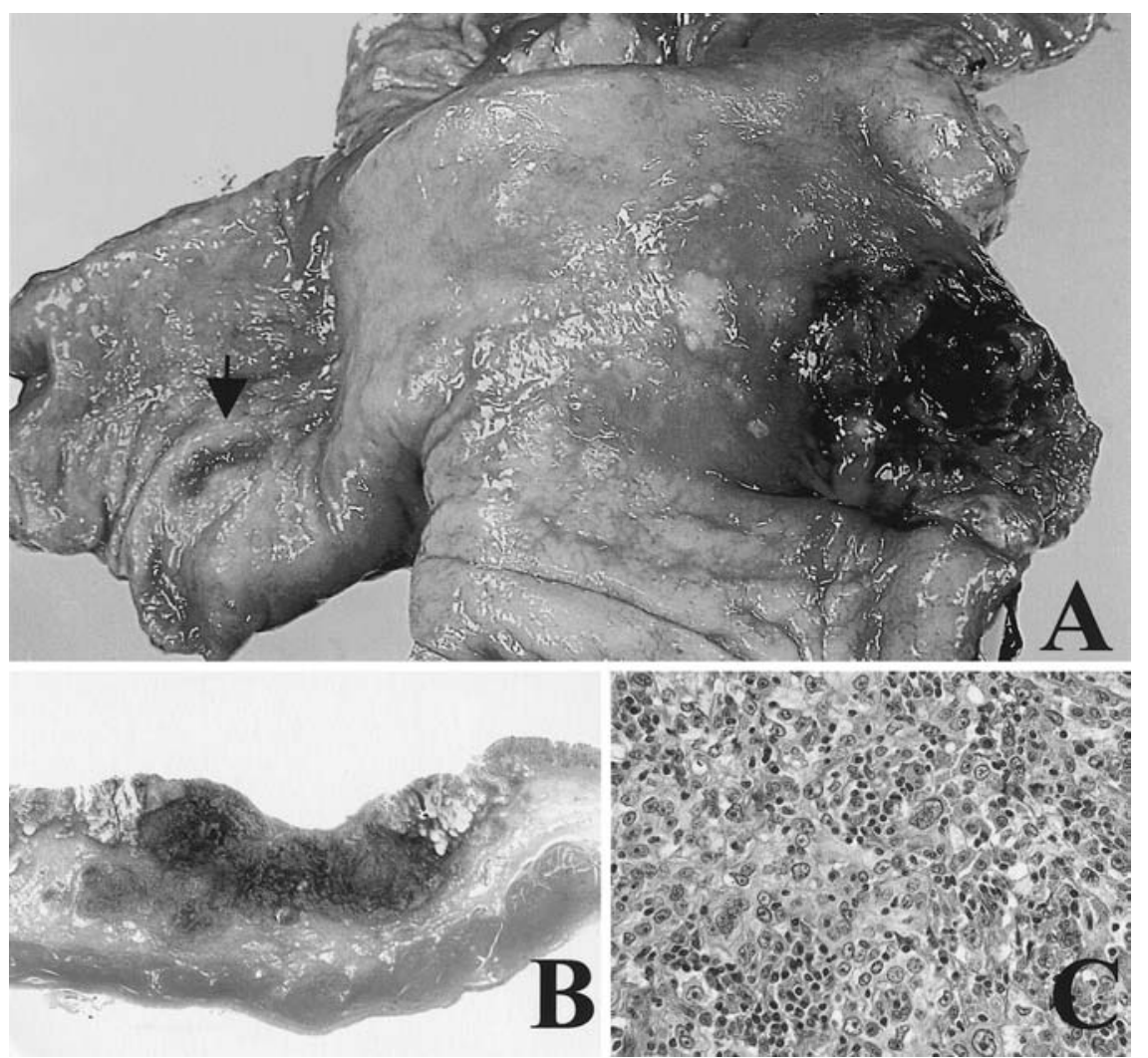

Fig. 1. A Macroscopic findings of the resected specimen. A type II c cancer was present in the lower stomach (black arrow) and disintegrating lymph node metastasis was exposed to mucosa in the upper stomach. B Histopathological findings of the type II c cancer. The cancer invaded within the submucosal layer. C Most of the tumor showed medullary proliferation of pleomorphic tumor cells, with no specific differentiation section were performed, and the degree of curability was $\mathrm{B}$ (relative curative resection), according to the Japanese classification of gastric carcinoma [2].

Macroscopically, a type II c cancer was present in the lower stomach, and disintegrating lymph node metastasis exposed to the mucosa was present in the upper stomach (Fig. 1A). Histopathologically, the primary II c lesion was within the submucosal layer (Fig. 1B), with marked lymphatic invasion. Lymph node metastases extended along the lesser curvature (No. 3) invading the gastric wall and pancreas, as well as along the common hepatic artery (No 8a,p) (pT1N3, stage IV) [2]. Most of the tumor showed medullary proliferation of pleomorphic tumor cells, with no specific differentiation (Fig. 1C), which led to a diagnosis of undifferentiated carcinoma. Although G-CSF immunostaining for gastric cancer and lymph node metastatic tissue was negative, the G-CSF m-RNA level (in frozen sections) showed a high increase, to 233.94 compared with 1.00 for the control (common gastric cancer tissue), using a real-time reverse transcriptase - polymerase chain reaction (RTPCR) method. After surgery, the fever subsided, and the leukocyte and G-CSF levels decreased to 13000/ $\mathrm{mm}^{3}$ and $35.6 \mathrm{pg} / \mathrm{ml}$, respectively. He had an uneventful course, and he was subsequently discharged. Chemotherapy with cisplatin plus TS-1 (S-1) [3] was administered, on an outpatient basis.

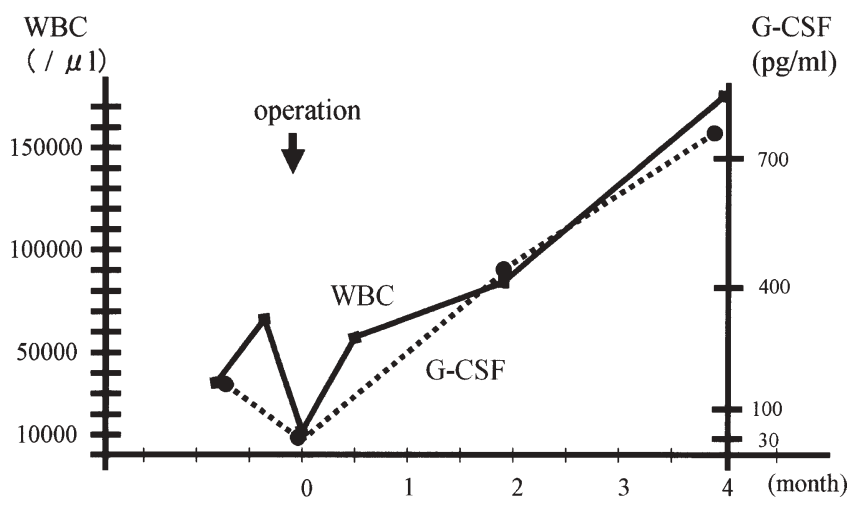

Fig. 2. (Changes in WBC (solid line) and granulocyte-colonystimulating factor $(G-C S F$; dotted line)

Two months after the surgery, the leukocyte and GCSF levels increased again, to $81000 / \mathrm{mm}^{3}$ (neutrophils more than $90 \%$ ) and $419 \mathrm{pg} / \mathrm{ml}$, respectively, and abdominal CT showed intraabdominal lymph node recurrence. Four months after the surgery, the leukocyte and G-CSF levels were markedly increased, to $169000 / \mathrm{mm}^{3}$ and $733 \mathrm{pg} / \mathrm{ml}$, respectively (Fig. 2). Chest X-ray revealed abnormal shadows in the left lung, raising suspicions of lung metastases (Fig. 3A). He then developed generalized seizures with a decreased level of con- 

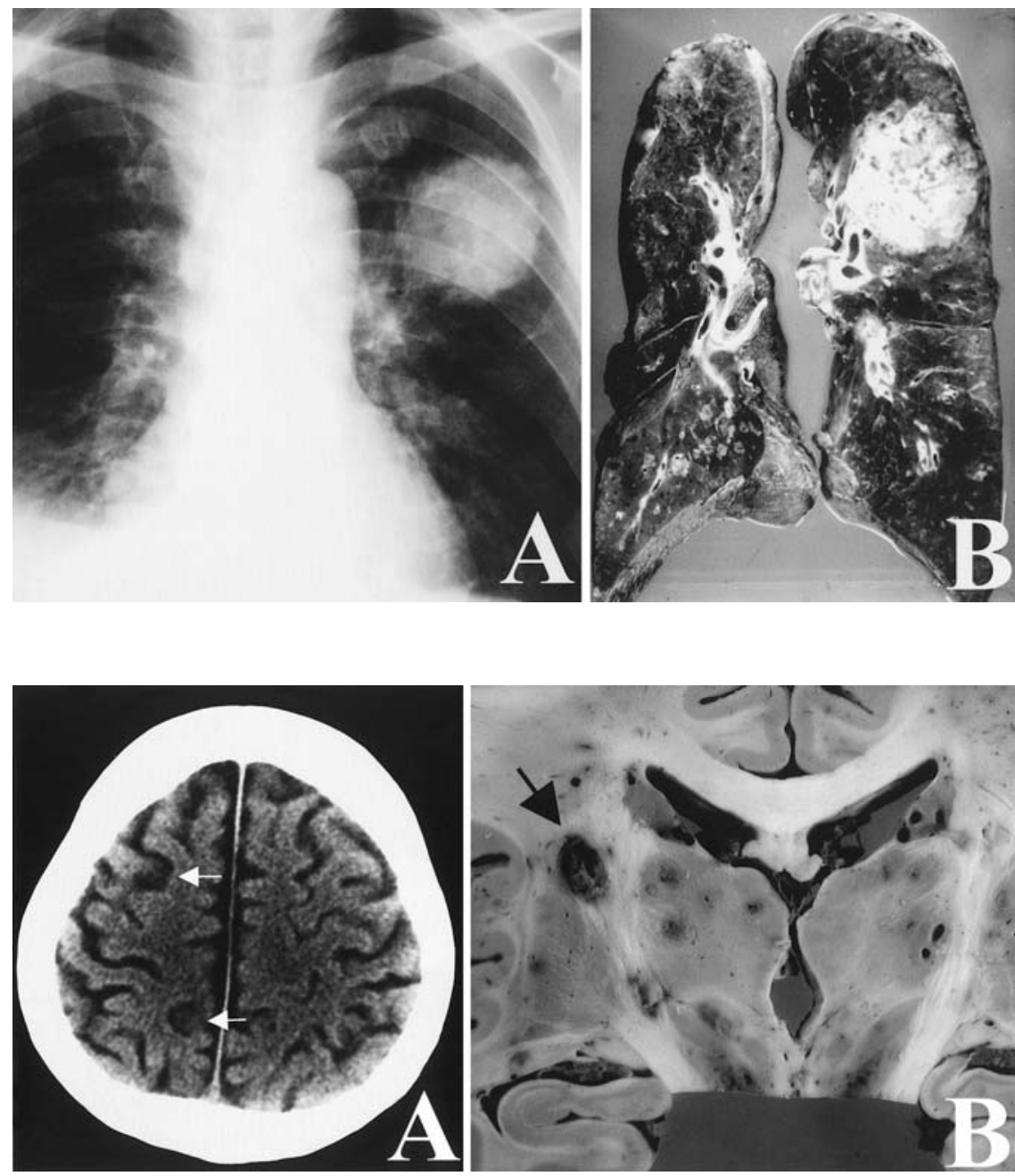

Fig. 3. A Chest X-ray showed abnormal shadows in the left lung, and metastases were suspected. B Lung tumors in the left superior lobe, and multiple white nodules in the bilateral inferior lobes, proved to be multiple abscesses, with marked infiltrations of neutrophils
Fig. 4. A Brain metastases (white arrows) were suspected on nonenhanced brain computed tomography (CT). B Multiple abscesses (black arrow) were recognized in the brain sciousness, and nonenhanced head CT scans suggested brain metastases (Fig. 4A). The patient also developed small skin abscesses on his face and abdominal wall, without any evidence of infectious bacteria. The patient died of multiple organ failure with drugresistant high fever and general seizures 4 months after the surgery.

Autopsy showed recurrence of undifferentiated carcinoma in the lymph nodes at the hepatic porta, around the abdominal aorta, and in the mediastinum. On histopathological examination, the lung tumors (including one that was $5 \mathrm{~cm}$ in size in the left superior lobe) and multiple white nodules in the bilateral inferior lobes, where metastases were suspected, proved to be multiple abscesses with marked infiltrations of neutrophils without cancer cells (Fig. 3B). Multiple abscesses, within $1 \mathrm{~cm}$ in size (which had caused the generalized seizures), were also recognized in the brain (Fig. 4B).

\section{Discussion}

G-CSF-producing tumors have been reported frequently in lung cancer [4], and sometimes in the digestive tract; for example, in the stomach, liver, gallbladder, and pancreas. Although more than 20 cases of G-CSF-producing gastric cancer, including ours, have been reported, mainly from Japanese institutes [1,5,6], little is known about the characteristic features associated with high levels of G-CSF and leukocytosis. The prognosis of G-CSF gastric cancer is extremely poor, and it was reported that many patients died approximately 2 to 6 months after diagnosis [1,5]. It is thought that, because these tumors are extremely malignant and grow very rapidly, they are already advanced at the time of detection, making the prognosis poor. The tumor in our patient was T1, and was highly malignant, with marked lymph node metastases of N3 and, rapid tumor enlargement after admission; the patient died 4 months after surgery in spite of receiving surgical resection and chemotherapy. 
Although the resected specimens were not positive for G-CSF by immunostaining, the following findings were consistent with G-CSF-producing gastric cancer: a highly elevated, quantifiable G-CSF m-RNA level in the tumor frozen sections (determined by real-time RT$\mathrm{PCR}$ ); tumor resection that resulted in transient decreases in the G-CSF and leukocyte levels to within a near-normal range; and recurrence of cancer that was associated with a simultaneous increase in G-CSF and neutrophil levels. Because G-CSF protein stays in the cell for only a short period and is then released, its detection has been reported to be difficult in some cases $[1,7,8]$. Recently, real-time RT-PCR has become a widely used method of quantifying cytokine levels in tissues and cells with high accuracy and sensitivity [9]. This method was very helpful in clarifying the present case as a G-CSF-producing tumor.

In our patient, the leukocyte levels increased to extremely high levels, up to $169000 / \mathrm{mm}^{3}$, when the recurrence became marked and there was formation of lung and brain abscesses. Metastases to the lung and brain were strongly suspected, but histopathological examination at autopsy confirmed that there were no cancer cells in the lung or brain, and demonstrated only neutrophil infiltrates, mimicking abscesses. As for the previously reported differential diagnosis of abscesses and G-CSF-producing tumors, there was a report of GCSF-producing glioblastoma mimicking brain abscess, proved by subtotal extirpation of the tumor [10].

With regard to whether or not markedly elevated GCSF causes the formation of abscesses, bullous pyoderma gangrenosum was reported to occur after G-CSF administration in a patient with lung cancer and neutropenic complications during anti-cancer chemotherapy [11]. Perianal and apical tooth abscesses, respectively, manifested in two healthy donors who received G-CSF for stem-cell mobilization [12]. The formation of these abscesses indicates the possible adverse effects of GCSF leading to neutrophil activation [13]. We speculate that the hypersecretion of G-CSF from the aggressive tumor in our patient resulted in neutrophil infiltrates forming abscesses in the skin, lung, and brain. Because no G-CSF-producing tumors have been reported to cause aseptic abscesses due to marked leukocytosis, as occurred in our patient, further studies of similar cases are necessary for the confirmation of this speculation.

\section{References}

1. Akutsu Y, Endo M, Hoshino T, Kuboshima M, Kagaya A, Tsunoda Y, et al. A case of granulocyte-colony stimulating factorproducing gastric cancer (in Japanese with English abstract). Jpn J Gastroenterol Surg 2003;36:1514-19.

2. Japanese Gastric Cancer Association. Japanese classification of gastric carcinoma. 2nd English ed. Gastric Cancer 1998;1:1024.

3. Koizumi W, Tanabe S, Saigenji K, Ohtsu A, Boku N, Nagashima F, et al. Phase I/II study of S-1 combined with cisplatin in patients with advanced gastric cancer. Br J Cancer 2003;89:220712.

4. Asano S, Urabe A, Okabe T, Sato N, Kondo Y, Ueyama Y, et al. Demonstration of granulopoietic factors in the plasma of nude mice with human lung cancer and in the tumor. Blood 1977;49: $845-52$.

5. Obara T, Ito Y, Kodama T, Fujimoto Y, Mizogichi H, Oshimi K, et al. A case of gastric carcinoma associated with excessive granulocytosis. Production of a colony-stimulating factor by the tumor. Cancer 1985;56:782-8.

6. Baba M, Hasegawa H, Nakayabu M, Shimizu S, Suzuki S, Kamada N, et al. Establishment and characteristic of a gastric cancer cell line (HuGC-OOHIRA) producing high level of G-CSF, GM-CSF, and IL6: the presence of autocrine growth control by G-CSF. Am J Hematol 1995;49:207-15.

7. Nomura H, Imazeki I, Oheda M, Kubota N, Tamura M, Ono M, et al. Purification and characterization of human granulocyte colony stimulating factor (G-CSF). EMBO J 1986;5:871-6.

8. Shimamura K, Fujimoto J, Hata J, Akatsuka A, Ueyama Y, Watanabe T, et al. Establishment of specific monoclonal antibodies against recombinant human granulocyte-stimulating factor (hG-CSF) and their application for immunoperoxidase staining of paraffin-embedded sections. J Histochem Cytochem 1990;38:2836.

9. Overbergh L, Giulietti A, Valckx D, Decallonne B, Bouillon R, Mathieu C. The use of real time reverse transcriptase PCR for the quantification of cytokine gene expression. J Biomol Tech 2003; 14:33-43.

10. Hintzen RQ, Voormolen J, Sonneveld P, Van Duinen SG. Glioblastoma causing granulocytosis by secretion of granulocytecolony-stimulating factor. Neurology 2000;54:259-61.

11. Ross HJ, Moy LA, Kaplan R, Figlin RA. Bullous pyoderma gangrenosa after granulocyte colony-stimulating factor treatment. Cancer 1991;68:441-3.

12. Hilbe W, Nussbaumer W, Bonatti H, Thaler J, Niederwieser D, Nachbaur D. Unusual adverse events following peripheral blood stem cell (PBSC) mobilization using granulocyte colony stimulating factor (G-CSF) in healthy donors. Bone Marrow Transplant 2000;26:811-3.

13. Spiekermann K, Roesler J, Emmendoerffer A, Elser J, Welte K. Functional features of neutrophils induced by G-CSF and GM-CSF treatment: differential effects and clinical implications. Leukemia 1997;11:466-78. 